\title{
Ultra-high pressure treatment controls in vitro fecal fermentation rate of insoluble dietary fiber from Rosa Roxburghii Tratt pomace and induces butyrogenic shifts in microbiota composition
}

Shaokang Wang a, b, Jie Xia a , Kim De Paepe b , Bin Zhang a, c, d, Xiong Fu a, c, d, Qiang Huang a, c, d *, Tom Van de Wiele ${ }^{b}$

${ }^{a}$ School of Food Science and Engineering, Guangdong Province Key Laboratory for Green Processing of Natural Products and Product Safety, Engineering Research Center of Starch and Vegetable Protein Processing Ministry of Education, South China University of Technology, Guangzhou 510640, China

${ }^{\mathrm{b}}$ Center for Microbial Ecology and Technology (CMET), Department of Biotechnology, Faculty of Bioscience Engineering, Ghent University, Coupure Links 653, 9000, Ghent, Belgium

c Sino-Singapore International Research Institute, Guangzhou 510555, China

${ }^{d}$ Overseas Expertise Introduction Center for Discipline Innovation of Food Nutrition and Human Health (111 Center), Guangzhou 510640, China

* Corresponding author

Tel.: +86 208711 3845; fax: +862087113848.

Email: qiangh@scut.edu.cn (Q. Huang) 


\section{Supporting Information}

Table S1. Annotation of the top 20 ASVs based on Greengenes database version 13.8.

\begin{tabular}{ccc}
\hline ASV number & ASV ID & Species name \\
\hline ASV1 & ee1c8b2b3c29b4f81d05d0ad3f51a975 & Gemmiger formicilis \\
ASV2 & 85ef2b44e4461455630addc2e6d0aad83 & Bacteroides uniformis \\
ASV3 & 6c3625df0fa5b45cdfca8e5d1912b010 & unclassified Ruminococcaceae \\
ASV4 & b0bca208e15ebf1c2fffcdc93a21a23b & Faecalibacterium prausnitzii \\
ASV5 & b44a606395e4d535902f895915dad9809 & unidentified Ruminococcus \\
ASV6 & 16579865380f4c8760057fd859c956dd & Akkermansia muciniphila \\
ASV7 & 45e7723852a11d4ebaal59996e77eced & unclassified Roseburia \\
ASV8 & 0dbc3b253d82ba703c41675b699b4d06 & unidentified Coprococcus \\
ASV9 & 0578f7d1751f553c3aab849bd40falac & unidentified Phascolarctobacterium \\
ASV10 & a243024683a23ca15e79735dd4e96da5 & unidentified Dialister \\
ASV11 & e3deb047ba5a 93b297efe94f72e90494 & Streptococcus luteciae \\
ASV12 & 7e4239f642d2d5f73eca9ccabfa418f4 & Coprococcus eutactus \\
ASV13 & 4ec4a134e420937ba0579397330858dd & unidentified Blautia \\
ASV14 & 8970b431812f6f3ad9b44d5be556a559 & unclassified Ruminococcaceae \\
ASV15 & 5036ca5512f20c4399126e591eb8281a & unidentified Ruminococcaceae \\
ASV16 & ac14f9aad13a287272b0bf0aa0310d57b & unidentified Bacteroides \\
ASV17 & 5fc91c8bc1ce5e0d1d669e1332ab69d2 & unidentified Bacteroides \\
ASV18 & ece065ac0 71d0fbc8db788fef42b34e1 & Clostridium clostridioforme \\
ASV19 & 1c0c03d5d81c2ff7169a2a529bced877 & unidentified Lachnospiraceae \\
ASV20 & 699a59715fe009887689479ee6db02f5 & unidentified Oscillospira \\
\hline
\end{tabular}


Figure S1. Chemical composition (A) and total (TDF) and insoluble (IDF) dietary fiber content (B) of Rosa roxburghii Tratt pomace (R) and $R$. roxburghii pomace water insoluble dietary fiber (RIDF).
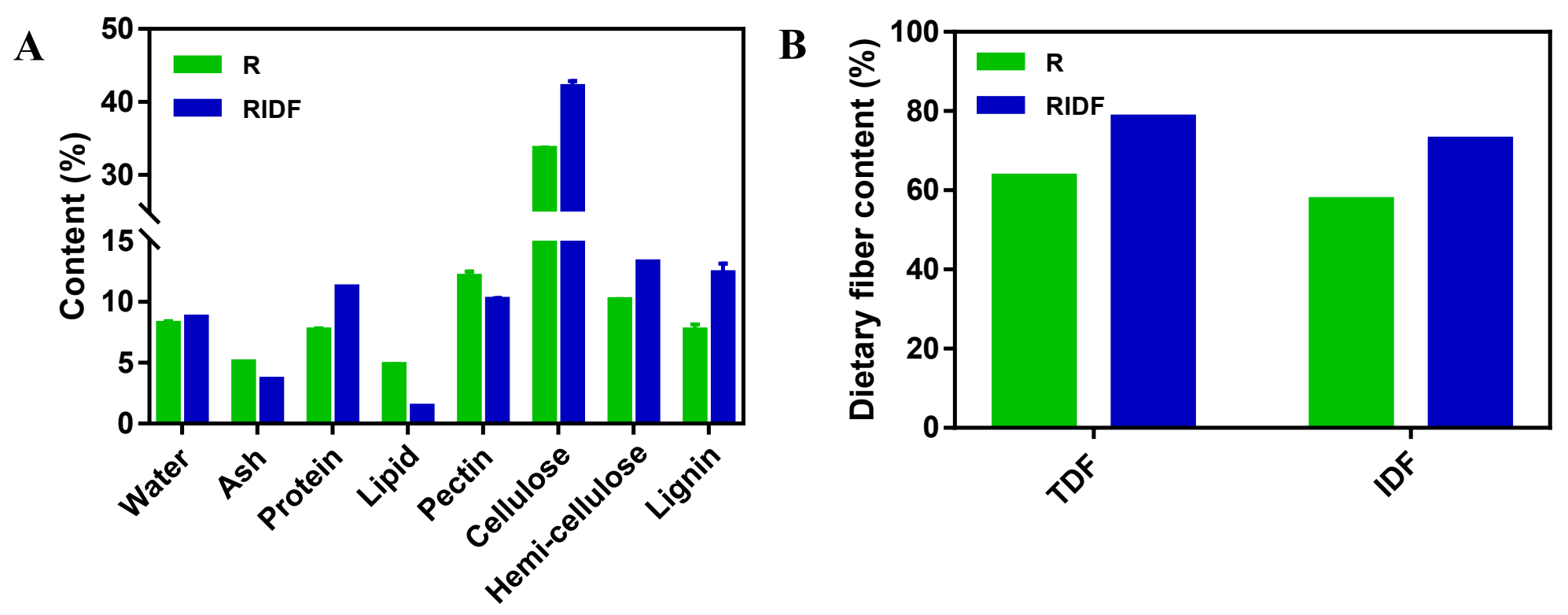
Figure S2. Phylum level changes in the microbiota composition after 0,12 and $24 \mathrm{~h}$ in vitro fecal fermentation of fructooligosaccharides (FOS), Rosa roxburghii Tratt pomace (R), $R$. roxburghii pomace water insoluble dietary fiber (RIDF) and ultra-high pressure treated RIDF in $90 \mathrm{MPa}$ (RIDF-90). 


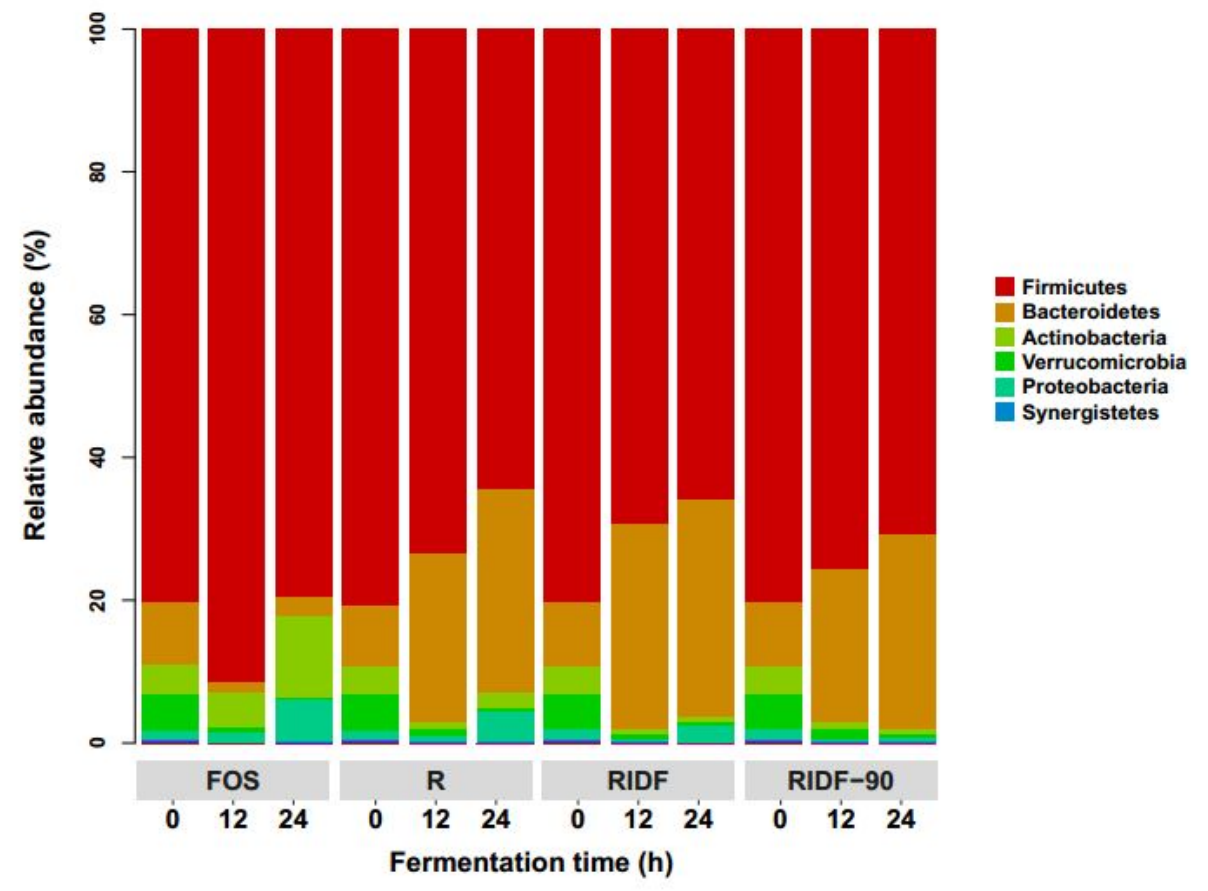

